Corresponding authors: daniel.koboldt@ nationwidechildrens.org; peter. white@nationwidechildrens.org

(c) 2018 Koboldt et al. This article is distributed under the terms of the Creative Commons

Attribution-NonCommercial

License, which permits reuse and redistribution, except for commercial purposes, provided that the original author and source are credited.

Ontology terms: absent speech; arthrogryposis multiplex congenita; decreased muscle mass; flexion contracture; fractures of the long bones; frontal bossing; generalized cerebral atrophy/hypoplasia; generalized muscle weakness; infantile spasms; prominent ear helix; relative macrocephaly; severe muscular hypotonia; skeletal myopathy; thick eyebrow

Published by Cold Spring Harbor Laboratory Press

doi: $10.1101 / \mathrm{mcs} . a 003160$

\section{In-frame de novo mutation in BICD2 in two patients with muscular atrophy and arthrogryposis}

\author{
Daniel C. Koboldt, ${ }^{1,2}$ Rama D. Kastury, ${ }^{3}$ Megan A. Waldrop, ${ }^{2,4}$ Benjamin J. Kelly, ${ }^{1}$ \\ Theresa Mihalic Mosher, ${ }^{1,2,5}$ Heather McLaughlin, ${ }^{6}$ Don Corsmeier, ${ }^{1}$ \\ Jonathan L. Slaughter, ${ }^{2,7}$ Kevin M. Flanigan, ${ }^{2,4,8}$ Kim L. McBride, ${ }^{2,5}$ \\ Lakshmi Mehta, ${ }^{3,9}$ Richard K. Wilson, ${ }^{1,2}$ and Peter White ${ }^{1,2}$ \\ ${ }^{1}$ Institute for Genomic Medicine, Nationwide Children's Hospital, Columbus, Ohio 43205, USA; ${ }^{2}$ Department \\ of Pediatrics, The Ohio State University, Columbus, Ohio 43210, USA; ${ }^{3}$ Department of Genetics and Genomic \\ Sciences, Icahn School of Medicine at Mount Sinai, New York, New York 10029, USA; ${ }^{4}$ Center for Gene \\ Therapy, Nationwide Children's Hospital, Columbus, Ohio 43205, USA; ${ }^{5}$ Division of Genetic and Genomic \\ Medicine, Nationwide Children's Hospital, Columbus, Ohio 43205, USA; ${ }^{6}$ GeneDx, Inc., Gaithersburg, \\ Maryland 20877, USA; ${ }^{7}$ Center for Perinatal Research and Division of Neonatology, Nationwide Children's \\ Hospital, Columbus, Ohio 43205, USA; ${ }^{8}$ Department of Neurology, The Ohio State University, Columbus, \\ Ohio 43210, USA; ${ }^{9}$ Department of Pediatrics, Icahn School of Medicine at Mount Sinai, New York, New York \\ 10029, USA
}

Abstract We describe two unrelated patients, a 12-yr-old female and a 6-yr-old male, with congenital contractures and severe congenital muscular atrophy. Exome and genome sequencing of the probands and their unaffected parents revealed that they have the same de novo deletion in BICD2 (c.1636_1638delAAT). The variant, which has never been reported, results in an in-frame 3-bp deletion and is predicted to cause loss of an evolutionarily conserved asparagine residue at position 546 in the protein. Missense mutations in $B I C D 2$ cause autosomal dominant spinal muscular atrophy, lower-extremity predominant 2 (SMALED2), a disease characterized by muscle weakness and arthrogryposis of early onset and slow progression. The p.Asn546del clusters with four pathogenic missense variants in a region that likely binds molecular motor KIF5A. Protein modeling suggests that removing the highly conserved asparagine residue alters BICD2 protein structure. Our findings support a broader phenotypic spectrum of BICD2 mutations that may include severe manifestations such as cerebral atrophy, seizures, dysmorphic facial features, and profound muscular atrophy.

[Supplemental material is available for this article.]

\section{CASE PRESENTATION}

Here we report two unrelated patients with muscular atrophy and arthrogryposis who ultimately were found to have the same molecular diagnosis. Their clinical features are compared in Table 1; additional clinical data are included in the supplement.

Patient 1 is a 12-yr-old girl with history of decreased fetal movement, bilateral femur fractures, and contractures of the ankles, digits, and wrists from birth. She required gastrostomy feeding and a tracheostomy, which was removed at age $8 \mathrm{yr}$. She had severe kyphoscoliosis and underwent multiple orthopedic procedures and spinal fusion surgery. On examination, she had relative macrocephaly with frontal bossing, thick eyebrows, prominent ears with 


\begin{tabular}{lcc}
\hline Table 1. Clinical features & & \\
\hline HPO term & Patient 1 -Female, 12 yr & Patient 2-Male, 6 yr \\
\hline Decreased fetal movement & + & + \\
Polyhydramnios & unk & + \\
Arthrogryposis multiplex congenita & + & + \\
Skeletal muscle atrophy & + & + \\
Muscle weakness & + & + \\
Recurrent fractures & + & + \\
Thoracolumbar kyphoscoliosis & + & + \\
Feeding difficulties & + & - \\
Macrocephaly & + & + \\
Frontal bossing & + & + \\
Abnormality of the ear & + & + \\
Open mouth & + & + \\
Downturned corners of mouth & + & + \\
Tapered fingers & + & + \\
EEG abnormality & + & + \\
Seizures & + & + \\
Cerebral cortical atrophy & + & + \\
Weak cry & unk & + \\
Absent speech & + & + \\
Left ventricular hypertrophy & + & + \\
Mitral regurgitation & + & + \\
& & +
\end{tabular}

Human Phenotype Ontology (HPO) terms are listed with an indication of whether each patient was positive (+), negative (-), or unknown (unk) for each feature.

hypoplastic outer helices and prominent anthelix, and mild midface hypoplasia with upturned nose and pointed nasal tip. Her extremities were thin, with muscle wasting and discoloration. She was nonverbal and nonambulatory with a history of "rolandic" seizures in the past. Brain MRI showed volume loss with thinning of the cortex.

Patient 2 is a 6-yr-old boy born at 38 wk gestation after a pregnancy noted to have severe polyhydramnios, fetal macrocephaly, and marked decreased fetal movement, particularly of the arms and legs. At birth he had dysmorphic features, bilateral symmetric facial weakness, micrognathia, weak cry, absent gag, and markedly decreased muscle bulk/tone with severe contractures (elbows, wrists, fingers, ankles in flexion, knees extended, hips internally rotated). Bone survey on day of life 1 showed right femur fracture. Neonatal brain MRI was consistent with very severe in utero hypoxic ischemic injury. Whole-body muscle MRI including the pelvis and thighs revealed marked absence of the musculature with fatty replacement (Supplemental Fig. 1). He developed intractable epilepsy and hydrocephalus requiring shunt placement at 6 mo of age. Follow-up brain MRI at $3.5 \mathrm{yr}$ showed near complete absence of cerebral white matter and cortex with subsequent enlargement of the ventricular system.

\section{VARIANT INTERPRETATION}

\section{Patient 1}

Whole-exome sequencing (WES) revealed a de novo, previously unreported, heterozygous deletion in BICD2 (Table 2). The c.1636_1638delAAT variant results in an in-frame 3-bp 
Table 2. Genomic findings and variant interpretation

\begin{tabular}{lcllll}
\hline Genomic location & HGVS cDNA & HGVS protein & Zygosity & Origin & Interpretation \\
\hline 9: 95481289 ATT/- & $\begin{array}{c}\text { NM_001003800: } \\
\text { c.1636_1638delAAT }\end{array}$ & $\begin{array}{c}\text { BICD2: p. } \\
\text { Asn546Del }\end{array}$ & Het & De novo & Likely pathogenic \\
\hline
\end{tabular}

Both Patient 1 and Patient 2 were found to have the same de novo mutation in BICD2. Genomic coordinates reflect build GRCh37.

deletion and is predicted to cause loss of an evolutionarily conserved Asparagine residue at position 546 in the protein, denoted as p.Asn546del. The c.1636_1638delAAT variant was not observed in large population cohorts (Lek et al. 2016). GeneDx interpreted the c.1636_1638delAAT variant as a likely pathogenic variant and submitted it to ClinVar in October 2016 (SCV000571877.3). The general assertion criteria for variant classification are publicly available on the GeneDx ClinVar submission page (http://www.ncbi.nlm.nih. gov/clinvar/submitters/26957//).

\section{Patient 2}

Whole-genome sequencing (WGS) revealed a heterozygous deletion in BICD2 (Table 2), which we interpreted according to ACMG guidelines (Richards et al. 2015). Sanger sequencing of the patient and both parents confirmed its de novo status. The c.1636_1638delAAT variant has not observed in the gnomAD populations, and is predicted to cause a deletion of a highly conserved asparagine residue in a nonrepeat region. The ClinVar report from GeneDx of the same de novo mutation in a patient with similar features provided further supporting evidence. Nationwide Children's interpreted the variant as pathogenic and submitted it to ClinVar in April 2018 (SCV000715101.1).

The p.Asn546Del variant maps just outside one of the coiled-coil domains that harbor several reported BICD2 mutations (Fig. 1A), but within a region that (in mice) interacts with molecular motor kinesin-1 (Splinter et al. 2010). A nearby variant, p.Arg501Pro, appears to increase the binding affinity of the BICD2 protein for the dynein-dynamin complex (Oates et al. 2013). Protein modeling suggests that the p.Asn546del variant alters protein structure (Supplemental Fig. 2), and that the changes to secondary structure are more striking for the p.Asn546del than for nearby missense mutations (Supplemental Fig. 3).

\section{SUMMARY}

The BicD gene was discovered in Drosophila and named bicaudal D ("having two tails"), because mutant embryos showed abnormal body patterning in which the head, thorax, and anterior abdominal segments are replaced with a mirror image of the posterior body segments and tail (Bullock and Ish-Horowicz 2001). The two mammalian homologs of BicD (BICD1 and BICD2) encode motor adaptor proteins that interact with the dyneindynactin complex and facilitate transport of mRNAs as well as other cellular cargoes (Vazquez-Pianzola et al. 2017).

Heterozygous missense changes in BICD2 cause autosomal dominant spinal muscular atrophy with lower extremity predominance (SMALED2, MIM \#615290) (Neveling et al. 2013; Oates et al. 2013; Peeters et al. 2013), a disease characterized by early-childhood or congenital onset of muscle weakness and atrophy. To date, 11 pathogenic missense variants 
A

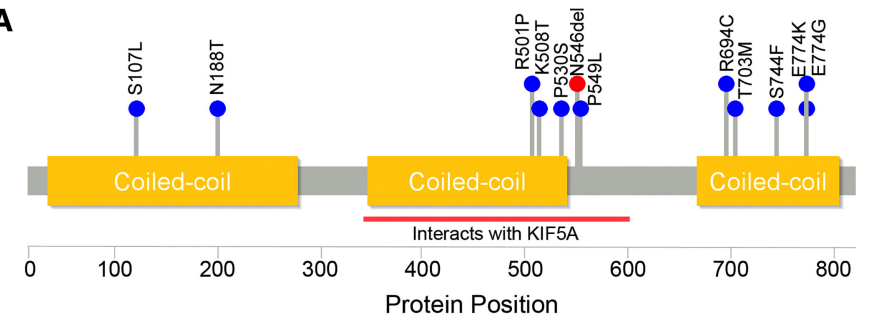

B

\section{UCSC Genome Browser on Human Feb. 2009 (GRCh37/hg19) Assembly}

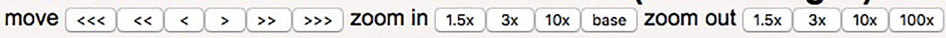

chr9:95,481,277-95,481,303 27 bp. enter position, gene symbol, HGVS or search terms go

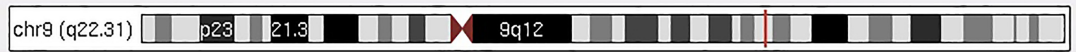

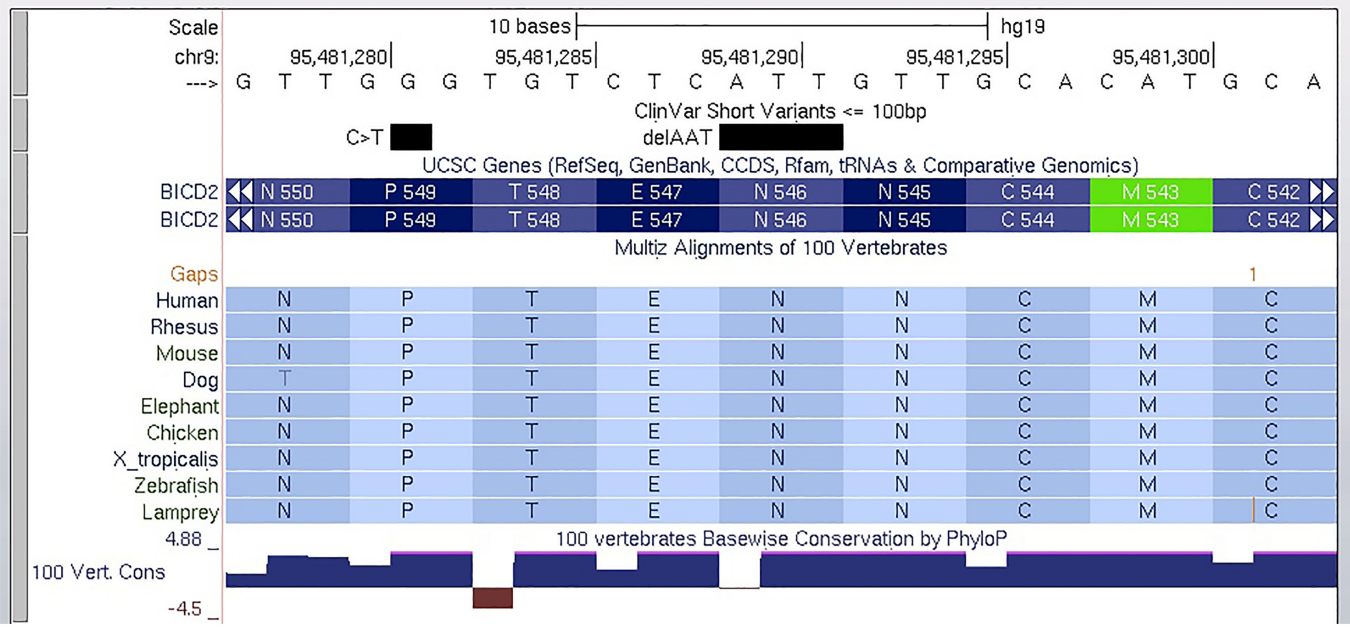

Figure 1. Location and conservation of the p.Asn546del variant. (A) Pathogenic and likely pathogenic variants reported in the ClinVar database as of July 3, 2018. Blue dots represent missense variants. The p.Asn542del variant is shown in red. BICD2 protein structure, coiled-coil domains (gold), and KIF5 interaction region (red bar) were taken from UniProtKB entry Q8TD16. (B) UCSC Genome Browser screenshot for the region harboring the p.Asn542del variant.

in BICD2 have been reported to the ClinVar database (Fig. 1A). The c.1636_1638delAAT variant is the only reported in-frame deletion in ClinVar; however, during preparation of this manuscript, Trimouille et al. (2018) published an in-frame deletion segregating in a family with variable disease severity.

There is a growing appreciation of the wide phenotypic spectrum of BICD2 mutations, which can range from asymptomatic to lethal congenital manifestations (Ravenscroft et al. 2016; Storbeck et al. 2017). A study of a four-generation Australian kindred with dominant spinal muscular atrophy (Oates et al. 2012) found that the clinical presentation of this disease can vary widely even within a single family. The manifestations in our patients are at the severe end of the spectrum, including lack of ambulation, dysmorphic craniofacial features, seizures, and cerebral atrophy. Patient 2 in particular had a striking lack of skeletal muscle, although there was also evidence of ischemic brain injury by MRI that could be a contributing factor. Even so, the substantial clinical overlap between our two patients, and the fact that they share the same de novo mutation, support the p.Asn546del mutation as a likely molecular basis for disease. 
COLD SPRING HARBOR Molecular Case Studies
BICD2 indels cause atrophy with arthrogryposis

\section{METHODS}

\section{Patient 1}

Using genomic DNA from the proband and parents, the exonic regions and flanking splice junctions of the genome were captured using the Clinical Research Exome kit (Agilent Technologies) by genetic testing provider GeneDx. Massively parallel (NextGen) sequencing was performed using an Illumina system with 100-bp paired-end reads. Additional sequencing technology and variant interpretation protocols have been previously described (Tanaka et al. 2015).

\section{Patient 2}

The proband and his parents underwent WGS $(2 \times 100 \mathrm{bp})$ performed using the Illumina HiSeq 2500 instrument. Reads were mapped to the GRCh37 reference sequence and secondary data analysis was performed using Churchill (Kelly et al. 2015). Our approach to variant annotation and prioritization has already been described (Koboldt et al. 2018).

More details on the sequencing and analysis can be found in the Supplemental Analysis and Methods section. Sequencing metrics are provided in Supplemental Table 1. The mutation diagram in Figure 1A was generated with Lollipops v1.3.2 (Jay and Brouwer 2016) using information from UniProt (entry \#Q8TD16) and the ClinVar (Landrum et al. 2018) database (accessed March 22, 2018). Protein modeling was performed using the Phyre2 (Kelley et al. 2015) and RaptorX (Källberg et al. 2012) web servers.

\section{ADDITIONAL INFORMATION}

\section{Data Deposition and Access}

The variants and their interpretations have been submitted to the ClinVar database (ID \#422408) (https://www.ncbi.nlm.nih.gov/clinvar/). Submitted reports are available under accession numbers SCV000571877.3 (Patient 1) and SCV000715101.1 (Patient 2). Raw sequencing data was not deposited because of lack of patient consent.

\section{Ethics Statement}

For Patient 1, written consent for GeneDx exome sequencing was obtained as part of the patient's clinical evaluation at Mount Sinai Medical Center. For Patient 2, written consent was obtained enrolling subjects into a research protocol approved by the Institutional Review Board at Nationwide Children's Hospital (IRB11-00215 Study: Using Genome Sequencing to Identify Causes of Rare Birth Defects and Rare Disorders).

\section{Acknowledgments}

We thank the patients and their families for participation in this research.

Competing Interest Statement

The authors have declared no competing interest.

Received May 10, 2018; accepted in revised form July 23, 2018.

\section{Author Contributions}

All authors contributed to scientific discussion, variant interpretation, and manuscript review.

\section{Funding}

This work was supported by The Research Institute at Nationwide Children's Hospital. 


\section{REFERENCES}

Bullock SL, Ish-Horowicz D. 2001. Conserved signals and machinery for RNA transport in Drosophila oogenesis and embryogenesis. Nature 414: 611-616.

Jay JJ, Brouwer C. 2016. Lollipops in the clinic: information dense mutation plots for precision medicine. PLoS One 11: e0160519.

Källberg M, Wang H, Wang S, Peng J, Wang Z, Lu H, Xu J. 2012. Template-based protein structure modeling using the RaptorX web server. Nat Protoc 7: 1511-1522.

Kelley LA, Mezulis S, Yates CM, Wass MN, Sternberg MJ. 2015. The Phyre2 web portal for protein modeling, prediction and analysis. Nat Protoc 10: 845-858.

Kelly BJ, Fitch JR, Hu Y, Corsmeier DJ, Zhong H, Wetzel AN, Nordquist RD, Newsom DL, White P. 2015. Churchill: an ultra-fast, deterministic, highly scalable and balanced parallelization strategy for the discovery of human genetic variation in clinical and population-scale genomics. Genome Biol 16: 6.

Koboldt DC, Mihalic Mosher T, Kelly BJ, Sites E, Bartholomew D, Hickey SE, McBride K, Wilson RK, White P. 2018. A de novo nonsense mutation in ASXL3 shared by siblings with Bainbridge-Ropers syndrome. Cold Spring Harb Mol Case Stud 4: a002410.

Landrum MJ, Lee JM, Benson M, Brown GR, Chao C, Chitipiralla S, Gu B, Hart J, Hoffman D, Jang W, et al. 2018. ClinVar: improving access to variant interpretations and supporting evidence. Nucleic Acids Res 46: D1062-D1067.

Lek M, Karczewski KJ, Minikel EV, Samocha KE, Banks E, Fennell T, O’Donnell-Luria AH, Ware JS, Hill AJ, Cummings BB, et al. 2016. Analysis of protein-coding genetic variation in 60,706 humans. Nature 536: 285-291.

Neveling K, Martinez-Carrera LA, Hölker I, Heister A, Verrips A, Hosseini-Barkooie SM, Gilissen C, Vermeer S, Pennings M, Meijer R, et al. 2013. Mutations in BICD2, which encodes a golgin and important motor adaptor, cause congenital autosomal-dominant spinal muscular atrophy. Am J Hum Genet 92: 946-954.

Oates EC, Reddel S, Rodriguez ML, Gandolfo LC, Bahlo M, Hawke SH, Lamandé SR, Clarke NF, North KN. 2012. Autosomal dominant congenital spinal muscular atrophy: a true form of spinal muscular atrophy caused by early loss of anterior horn cells. Brain 135: 1714-1723.

Oates EC, Rossor AM, Hafezparast M, Gonzalez M, Speziani F, MacArthur DG, Lek M, Cottenie E, Scoto M, Foley AR, et al. 2013. Mutations in BICD2 cause dominant congenital spinal muscular atrophy and hereditary spastic paraplegia. Am J Hum Genet 92: 965-973.

Peeters K, Litvinenko I, Asselbergh B, Almeida-Souza L, Chamova T, Geuens T, Ydens E, Zimoń M, Irobi J, De Vriendt $E$, et al. 2013. Molecular defects in the motor adaptor BICD2 cause proximal spinal muscular atrophy with autosomal-dominant inheritance. Am J Hum Genet 92: 955-964.

Ravenscroft G, Di Donato N, Hahn G, Davis MR, Craven PD, Poke G, Neas KR, Neuhann TM, Dobyns WB, Laing NG. 2016. Recurrent de novo BICD2 mutation associated with arthrogryposis multiplex congenita and bilateral perisylvian polymicrogyria. Neuromuscul Disord 26: 744-748.

Richards S, Aziz N, Bale S, Bick D, Das S, Gastier-Foster J, Grody WW, Hegde M, Lyon E, Spector E, et al. 2015. Standards and guidelines for the interpretation of sequence variants: a joint consensus recommendation of the American College of Medical Genetics and Genomics and the Association for Molecular Pathology. Genet Med 17: 405-424.

Splinter D, Tanenbaum ME, Lindqvist A, Jaarsma D, Flotho A, Yu KL, Grigoriev I, Engelsma D, Haasdijk ED, Keijzer N, et al. 2010. Bicaudal D2, dynein, and kinesin-1 associate with nuclear pore complexes and regulate centrosome and nuclear positioning during mitotic entry. PLoS Biol 8: e1000350.

Storbeck M, Horsberg Eriksen B, Unger A, Hölker I, Aukrust I, Martínez-Carrera LA, Linke WA, Ferbert A, Heller $\mathrm{R}$, Vorgerd M, et al. 2017. Phenotypic extremes of BICD2-opathies: from lethal, congenital muscular atrophy with arthrogryposis to asymptomatic with subclinical features. Eur J Hum Genet 25: 1040-1048.

Tanaka AJ, Cho MT, Millan F, Juusola J, Retterer K, Joshi C, Niyazov D, Garnica A, Gratz E, Deardorff M, et al. 2015. Mutations in SPATA5 are associated with microcephaly, intellectual disability, seizures, and hearing loss. Am J Hum Genet 97: 457-464.

Trimouille A, Obre É, Banneau G, Durr A, Stevanin G, Clot F, Pennamen P, Perez JT, Bailly-Scappaticci C, Rouanet $M$, et al. 2018. An in-frame deletion in BICD2 associated with a non-progressive form of SMALED. Clin Neurol Neurosurg 166: 1-3.

Vazquez-Pianzola P, Schaller B, Colombo M, Beuchle D, Neuenschwander S, Marcil A, Bruggmann R, Suter B. 2017. The mRNA transportome of the BicD/Egl transport machinery. RNA Biol 14: 73-89. 


\section{COLD SPRING HARBOR Molecular Case Studies}

\section{In-frame de novo mutation in BICD2 in two patients with muscular atrophy and arthrogryposis}

Daniel C. Koboldt, Rama D. Kastury, Megan A. Waldrop, et al.

Cold Spring Harb Mol Case Stud 2018, 4: a003160 originally published online July 27, 2018 Access the most recent version at doi: $10.1101 / \mathrm{mcs}$.a003160

Supplementary http://molecularcasestudies.cshlp.org/content/suppl/2018/08/01/mcs.a003160.D
Material

References This article cites 19 articles, 1 of which can be accessed free at:

http://molecularcasestudies.cshlp.org/content/4/5/a003160.full.html\#ref-list-1

License This article is distributed under the terms of the Creative Commons Attribution-NonCommercial License, which permits reuse and redistribution, except for commercial purposes, provided that the original author and source are credited.

Email Alerting Receive free email alerts when new articles cite this article - sign up in the box at the Service top right corner of the article or click here. 